\title{
Preparative-scale synthesis of nonacene
}

\author{
Andrej Jančař́ik ${ }^{1,2 *}$, Jan Holec ${ }^{1}$, Yuuya Nagata ${ }^{3}$, Michal Šámal $^{2}$, Andre Gourdon ${ }^{*}$ \\ 1) CEMES-CNR, 29 Rue J. Marvig, 31055 Toulouse, France. \\ 2) Institute of Organic Chemistry and Biochemistry of the Czech Academy of Sciences, 16610 Prague 6, Czech \\ Republic. \\ 3) Japan Institute for Chemical Reaction Design and Discovery (WPI-ICReDD), Hokkaido University, Sapporo, \\ Hokkaido 001-0021, Japan. \\ *emails: andrej.jancarik@ cemes.fr, andre.gourdon@cemes.fr
}

\begin{abstract}
:
During the last years we have witnessed progressive evolution of preparation of acenes with length up to dodecacene by on-surface synthesis in UHV or generation of acenes up to decacene in solid matrices at low temperatures. While these protocols with very specific conditions produce the acenes in amount of few molecules, the strategies leading to the acenes in large quantities dawdle behind. Only recently and after 70 years of synthetic attempts, heptacene has been prepared in bulk phase. However, the preparative scale synthesis of higher homologues still remains a formidable challenge. Here we report the preparation and characterisation of nonacene and show its excellent thermal and in-time stability.
\end{abstract}

Over the last decade, particular attention has been brought to long unsubstituted acenes (longer than pentacene) from both experimental and theoretical points of view. ${ }^{1}$ The nature of their electronic structure such as gap stabilization ${ }^{2-4}$ and open shell singlet ground state for longer acenes is still actively discussed. ${ }^{5-9}$ Furthermore, longer acenes can be seen as the narrowest zig-zag graphene nanoribbons (ZGNR) and could display spin-polarized edge-states of interest for carbon-based spin electronics. ${ }^{10,11}$

However, acenes longer than pentacene are challenging to prepare and to handle. First, intermolecular $\pi-\pi$ stacking between these planar and rigid molecules rapidly limits their solubility as their sizes increase. Second, acenes possess only one Clar aromatic sextet spread over the whole skeleton, leading to a decrease of the HOMO-LUMO gap with an increasing number of benzene rings, and therefore to an increase of the chemical reactivity. Although photooxidation with molecular oxygen can be avoided by working under argon, rapid dimerization in solution even at low concentration might become a problematic limitation.

Several strategies have been pursued to prepare long acenes, all relying on the same concept: masked stable and soluble precursors are prepared and purified by standard in-solution chemistry techniques and, in a final step, the masking groups are removed in the solid state or at low temperature in very dilute conditions on surfaces or in matrices. In particular Neckers, Bettinger and coworkers have explored the photogeneration, in stabilizing matrices, of hexacene, ${ }^{12}$ heptacene ${ }^{13-16}$, octacene, nonacene ${ }^{17}$, and undecacene ${ }^{18}$ by photodecarbonylation of precursors comprising two bridging $\alpha$-diketone groups, following Yamada's concept ${ }^{19}$ using the Strating-Zwanenburg ${ }^{20}$ reaction. Long acenes can also be prepared by on-surface synthesis in Ultra High Vacuum (UHV) and observed at liquid helium temperature and their electronic structures mapped by Scanning Tunneling Spectroscopy (STS). Higher acenes up to undecacene have been obtained by deoxygenation of epoxides, ${ }^{4,21,22}$ dehydrogenation of 
partially saturated precursors, ${ }^{23,24}$ or thermal or photo decarbonylation of diketone adducts. ${ }^{25-}$ 27

However, both types of generation, in stabilizing matrices or on surfaces in UHV only give minute amounts of materials and cannot be used for macroscopic amounts of materials needed for applications. Indeed, the preparation of acenes longer than pentacene in a pure state is very recent despite 70 years of claims, ${ }^{28}$ and so far limited to hexacene and heptacene. In 2012, Chow and coworkers isolated hexacene by decarbonylation of a monoketone precursor in the solid state and its structure was determined by X-ray diffraction. ${ }^{29}$ They demonstrated that a field-effect transistor made with a single crystal of hexacene showed a hole mobility significantly higher than pentacene. In 2017, Bettinger et al. have reported the formation of heptacene in the solid state by thermal cycloreversion from a mixture of diheptacenes obtained in solution. ${ }^{30}$ More recently, we have also obtained heptacene and benzohexacene by cheletropic decarbonylation at moderate temperature, confirming the thermal stability of these higher acenes. ${ }^{31}$ The preparation of even longer acenes in bulk form has been indeed an attracting challenge since Clar's prediction in 196 claiming that the synthesis of octacene (and beyond) was a remote target. ${ }^{32}$ And in a recent review, C. Tönshoff and H. F. Bettinger conclude that "It is not even clear if acenes larger than that of heptacene can exist outside the special environment provided by matrix isolation or on-surface synthesis". 33

To answer this question, we present here the synthesis of nonacene $\mathbf{1}$ and demonstrate its surprising stability.

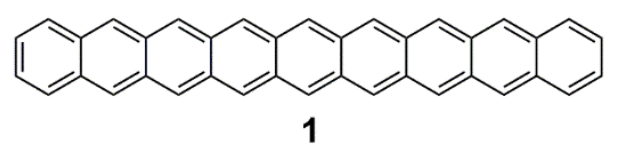

Fig. 1. Molecular structure of nonacene 1.

\section{Results and discussion}

As nonacene was expected to be highly insoluble and reactive, our strategy is based on pure soluble and chemically stable masked nonacene, that could be deprotected quantitatively by heating at medium temperatures in the solid state. In previous contributions, we have shown ${ }^{31,34}$ that 7,7-dimethoxy-2,3,5,6-tetramethylenebicyclo[2.2.1]-heptane (further in the text as tetraene) (scheme 1), which can be prepared at the tens of grams scale, ${ }^{35}$ can undergo successive Diels-Alder reaction with arynes to provide non-planar, soluble, and not fully delocalized acene precursors. In these compounds, one of the benzene rings is bridged by a dimethyl ketal group.

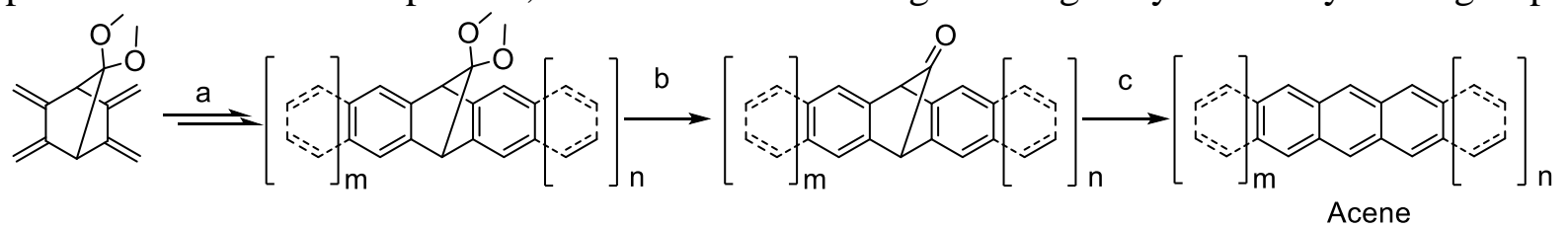

Scheme 1. Schematic strategy of the preparation of various acenes starting from 7,7-dimethoxy-2,3,5,6tetramethylenebicyclo[2.2.1]-heptane. Diels-Alder addition (a) with arynes, followed by aromatization gives a non-planar bridging dimethylketal, which can be deprotected (b) to yield a polyaromatic precursor bridged by a carbonyl group. (c) Solid state thermal or photochemical decarbonylation gives the acene with only carbon monoxide as by-product.

Cleavage of this ketal yields the corresponding polyaromatic acene precursors comprising norbornadiene-7-one moieties, mentioned simply as ketone precursors in this report. These compounds are at least partially soluble, chemically stable and can be purified by standard insolution techniques such as chromatography or recrystallization. They are stable enough to be stored in the dark for long period of time. The final step is a thermal or photochemical cheletropic decarbonylation in the solid state yielding the corresponding acenes in quantitative yields without any non-volatile by-products. It has been shown that this method of 
decarbonylation was very effective to prepare sensitive high quality materials, such as pentacene and hexacene, ${ }^{36}$ for opto-electronic devices.

Our exploration of nonacene $\mathbf{1}$ commenced with the synthesis of such a carbonylated precursor $\mathbf{7 a} / \mathbf{7 b}$ which can be easily transformed in the solid-state form to the nonacene by simple heating.

The synthesis of the precursors $\mathbf{7 a / 7} \mathbf{b}$ leading to nonacene $\mathbf{1}$ follows the route shown in scheme 2 with four synthetic steps starting from the diene 2 , which can be prepared in two steps from the tetraene shown in scheme 1 above. ${ }^{34}$<smiles>C=C1C(=C)C2(OC)OC3(COCCO3)C1c1cc3ccccc3cc12</smiles>

2

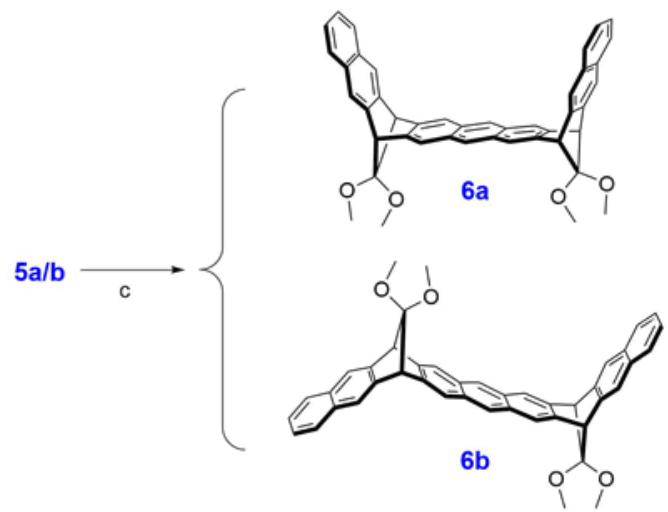

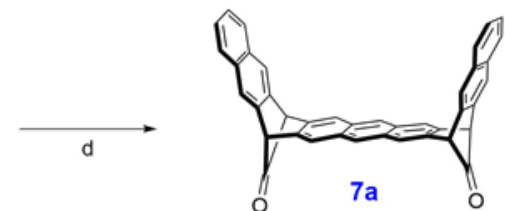

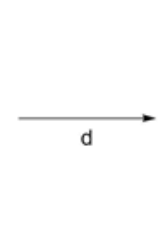

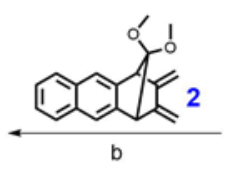

4

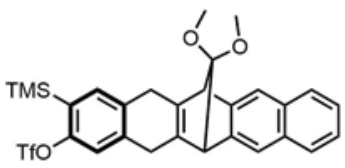

Scheme 2. Synthesis of nonacene 1: a) CsF, acetonitrile/THF (4:1), room temperature, 16 h., $62 \%$ (anti : syn 1:2); b) CsF, acetonitrile/THF (5:1), room temperature, 16 h., $98 \%$ (anti : syn 1:2); c) DDQ, toluene, room temperature, $4 \mathrm{~h} .94 \%$; d) TMSI, room temperature, $24 \mathrm{~h}$., 94\% for $7 \boldsymbol{a}$ and $48 \mathrm{~h}$., $95 \%$ for $7 \boldsymbol{b}$; e) neat $350{ }^{\circ} \mathrm{C}, 20$ min., quant.

The key reaction of the synthetic sequence is the double Diels-Alder reaction of the diene 2 and the in situ generated bis(aryne) obtained by fluoride-induced decomposition of 2,5bis(trimethylsilyl)-1,4-phenylene bis(trifluoromethanesulfonate) $\mathbf{3}^{37,38,39}$ Alternatively, the Diels-Alder reaction can be carried out from the aryne precursor 4 (synthesized from the same tetraene in three steps in 33\% yield), with the diene 2 . The formed mixture of these two isomers syn and anti 5a/5b can be easily separated on silica gel. As expected the NMR spectra of the two isomers are almost identical and owing to their symmetry and to the distance between the ketal groups, it is not possible to assign their structures by proton and carbon NMR spectroscopy. Fortunately, slow evaporation of a solution of the isomer $\mathbf{5 b}$ in a mixture of solvents (hexane/EtOAc) provided suitable crystals for X-ray analysis (Fig. 1). 


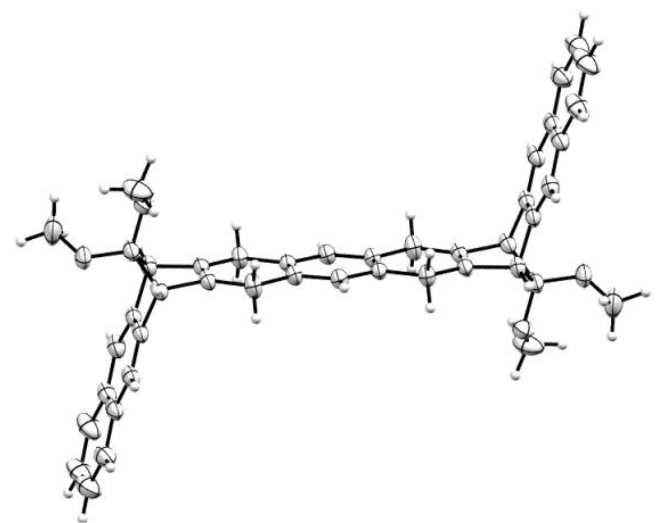

Fig. 1. Ortep representation of isomer $\mathbf{5 b}$

It shows that the two naphthalene ends of the anti-isomer $\mathbf{5 b}$ are perfectly parallel to each other and the angle between naphthalene and anthracene units is $105.5^{\circ}$.

Surprisingly, these products $\mathbf{5 a}$ and $\mathbf{5 b}$ were formed in the ratio $1: 2$ in favour of synisomer 5a. Indeed, considering the distance between the reactive sites, a statistical ratio 1:1 was initially expected. In order to investigate the stereoselectivity of the Diels-Alder reactions between the diene $\mathbf{2}$ and the benzyne compounds in acetonitrile, their transition states (TS) were determined by using density functional theory (DFT). ${ }^{40}$ By using Gaussian 16, Revision C.01, ${ }^{40}$ the geometries of TS were optimized with QST3 method at the B3LYP/6-31+G(d,p) level of theory with the polarizable continuum model (PCM) to include solvent effects (acetonitrile). Subsequently, single point energy calculations of TS were calculated at the M06-2X/6$31+\mathrm{G}(\mathrm{d}, \mathrm{p})$ level of theory with PCM (acetonitrile). ${ }^{41}$ Based on the activation energy determined by the calculations, anti/syn ratio was estimated to $1 / 1.64$, which is consistent with the observed stereoselectivity in the experiment (anti/syn $=1 / 2$ ) (See supporting information for details).

The isomers $\mathbf{5 a}$ and $\mathbf{5 b}$ underwent smooth aromatization by DDQ at room temperature in almost quantitative yield. Then, the two dimethylketal groups of $\mathbf{6 a / 6} \mathbf{b}$ were cleaved by trimethylsilyl iodide, which afforded the corresponding carbonyl isomers $\mathbf{7 a}$ and $\mathbf{7 b}$ in $94 \%$ and 95\% yield, respectively. The anti-isomer $\mathbf{7 b}$ is less soluble in many organic solvents than its counterpart 7a, likely due to the ability to pack in quasi one-dimensional chains with efficient $\pi-\pi$ stacking, which is not the case for U-shape isomer 7a. Both isomers are colourless chemically stable compounds.

Decarbonylation of $\mathbf{7 a} / \mathbf{7 b}$ in the solid state can be followed by thermal gravimetric analysis (TGA) as shown in Fig. 2.

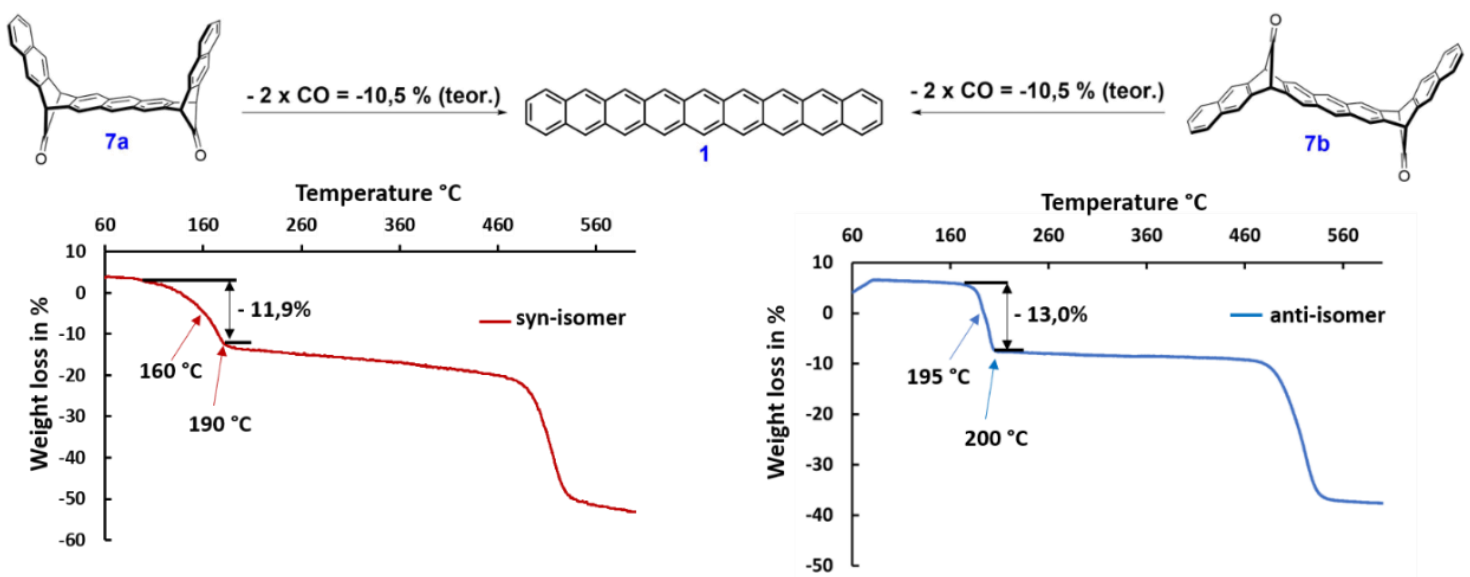
$10.5 \%)$

Fig. 2. TGA thermograms of 7a/7b showing the weight loss of two CO groups (ca 12 and 13\%; calcd. 
A weight loss of $11.9 \%$ for syn-isomer and $13.0 \%$ for anti-isomer (calcd 10.5\%) correspond to the loss of two carbonyl groups per molecule. The full decarbonylation occurred below $190^{\circ} \mathrm{C}$ in a one step process for both isomers. However, in the case of syn-isomer $7 \mathbf{a}$, the TGA thermogram consists of a gradual weight loss starting at about $60^{\circ} \mathrm{C}$. This can be explained by a lower thermal stability compared to the anti-isomer (starting around $180^{\circ} \mathrm{C}$ ). The decarbonylation is accompanied by a colour change from white to anthracite and the formation of nonacene. Under these conditions, nonacene is surprisingly thermally stable up to almost $500^{\circ} \mathrm{C}$. The formation of nonacene 1 by loss of two carbonyl groups is also evident during the high-resolution ESI MS measurement of 7a/7b in which only a peak at $\mathrm{m} / \mathrm{z} 478.1731$ from $\mathbf{7 a}$ or 478.1727 from $\mathbf{7 b}$ corresponding to the formula $\mathrm{C}_{38} \mathrm{H}_{22}$ (calcd m/z: 478.1722) was recorded.

The decarbonylation process was also followed by FTIR experiments (Fig. 3), where the stretching vibration of carbonyl peak at $1780 \mathrm{~cm}^{-1}$ disappear after heating the carbonyl precursor in $\mathrm{KBr}$ pellet.

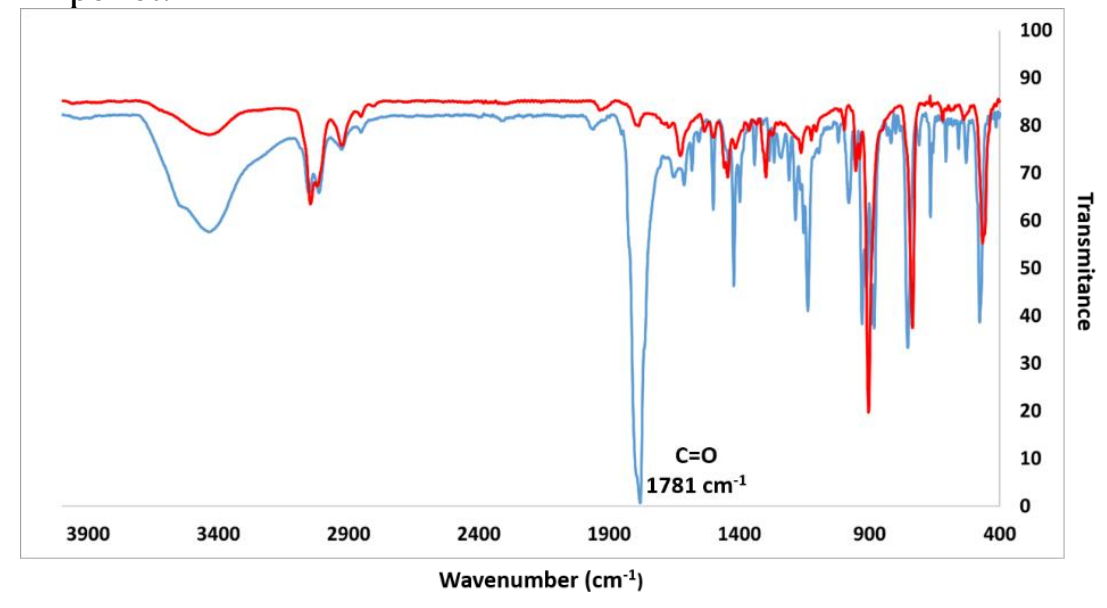

Fig. 3. FTIR spectra (KBr pellets) of the carbonylated precursors $7 \boldsymbol{b}$ (in blue) with a strong $C O$ peak at $1781 \mathrm{~cm}^{-1}$ and of the resulting nonacene 1 (in red) after $1 \mathrm{~min}$. heating at $350^{\circ} \mathrm{C}$ in glovebox.

This transformation has been also followed by solid state cross-polarization magic angle spinning (CP-MAS) NMR spectroscopy. The spectrum of the precursor 7a shows (Fig. 4) three groups of signals, one at $57 \mathrm{ppm}$ (bridgehead $\mathrm{sp}^{3}$ carbons), a complex peak at 120-137 ppm (aromatic carbons) and the carbonyl carbons at 193 and $198 \mathrm{ppm}$. Despite the symmetry of the molecule, the different environments in the solid state of the two carbonyl groups is a cause of these two signals. After heating the sample for 20 minutes at $200^{\circ} \mathrm{C}$ under inert atmosphere, the carbonyl signals disappear and aromatic region get narrower (Fig. 4). However, a smaller and broader $\mathrm{sp}^{3}$ signal at $54 \mathrm{ppm}$ remains. We attribute this peak to partial dimerization/polymerization by of the decarbonylated compound. Upon heating at higher temperatures, this peak decreases whereas the peak attributed to the aromatic carbons gets narrower with a decrease of the shoulder at $137 \mathrm{ppm}$. The evolution of the CPMAS spectrum of the isomer $\mathbf{7 b}$ is very similar (see SI). 


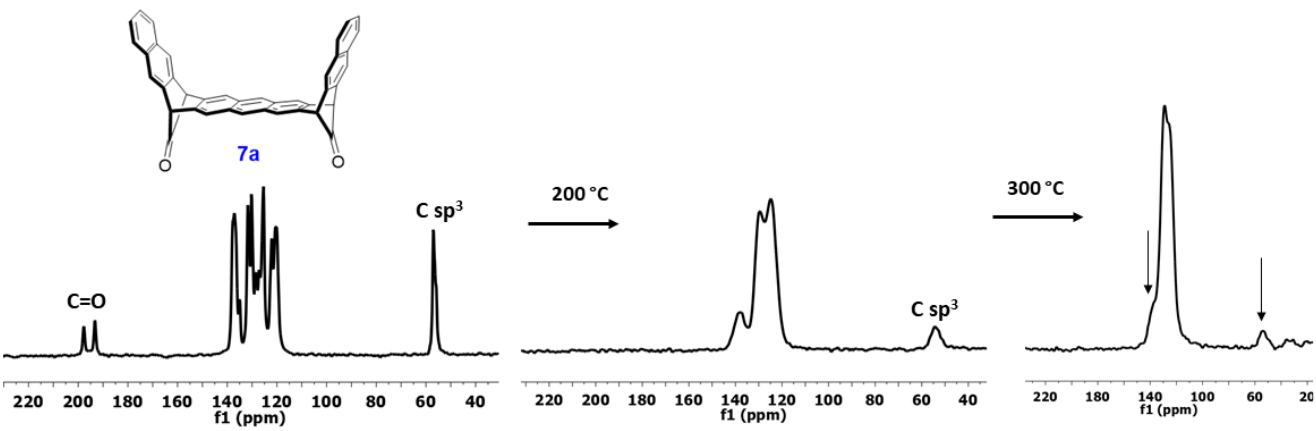

Fig. 4 : Evolution of the CPMAS ${ }^{13} C N M R$ spectra of 7 a (left) in function of the temperature.

This behaviour is very reminiscent to that of heptacene. ${ }^{30}$ The latter molecule dimerizes in solution during its preparation, but cycloreversion to heptacene of the two dimers has been observed by heating for $12 \mathrm{~min}$ at $300^{\circ} \mathrm{C}$ in the solid state. However, after several weeks, some of the heptacene reacted back to the dimers.

Based on the TGA experiment, the formed nonacene is surprisingly stable almost up to $500^{\circ} \mathrm{C}$, which allows to realize the extrusion of carbonyl groups at much higher temperature. In a new experiment we carried out the decarbonylation of the precursors $7 \mathbf{a} / 7 \mathbf{b}$ at $350^{\circ} \mathrm{C}$ for 20 min (Fig. 6). Gratifyingly, the decarbonylation process was much cleaner with only a sharp doublet in aromatic region without any signs of dimerization. Keeping these samples in the NMR rotor at room temperature in a glove box for 2 months did not lead to any degradation or dimerization of nonacene $\mathbf{1}$ suggesting that the nonacene prepared under these conditions is stable.

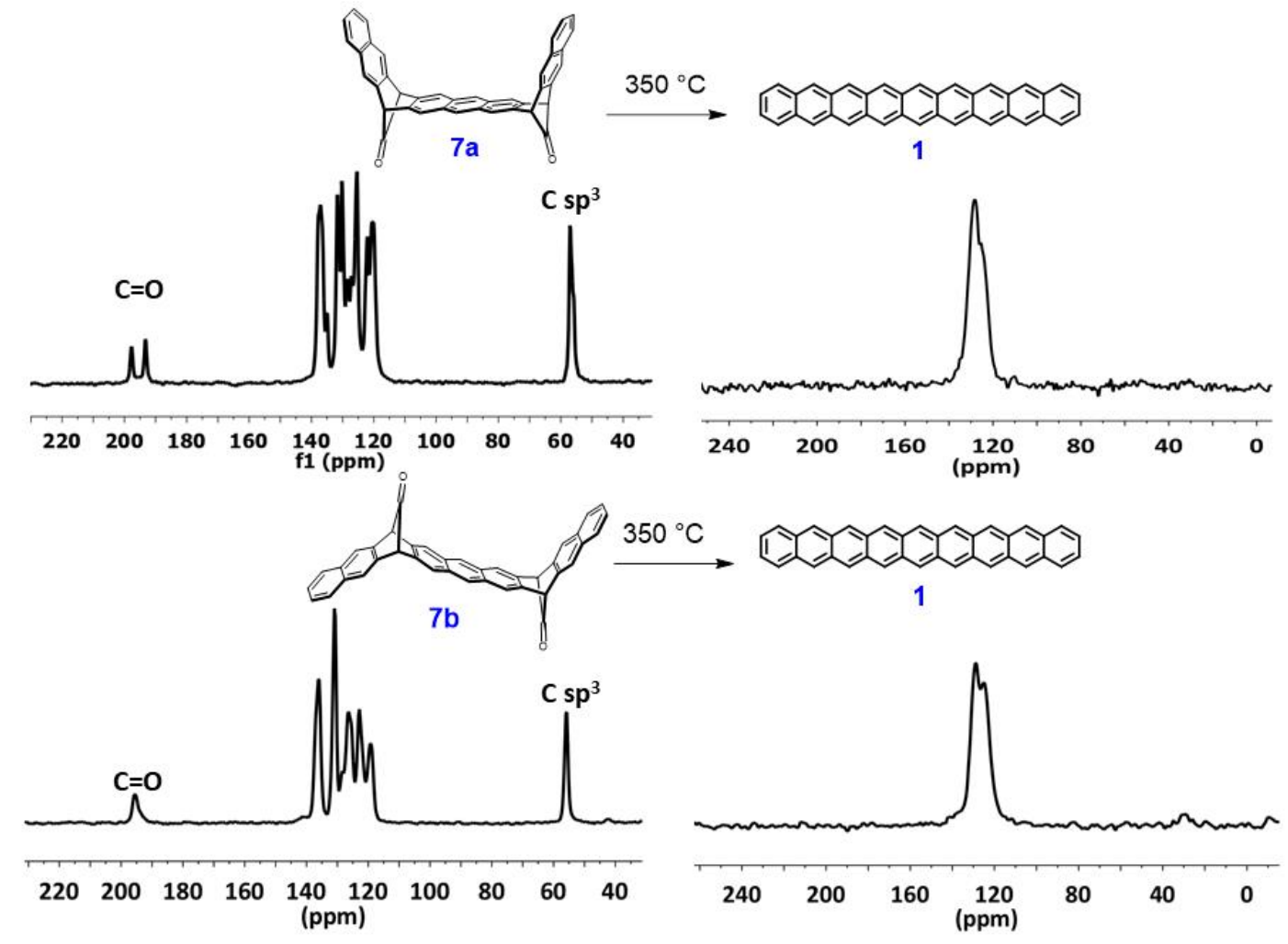

Fig. 6. Evolution of the CPMAS spectra of $7 \mathrm{a} / 7 \mathrm{~b}$ by decarbonylation at $350^{\circ} \mathrm{C}$ for 20 min to form the nonacene 1 . 
Alternatively, nonacene $\mathbf{1}$ can also be obtained by decarbonylation in a solvent at high temperature. For instance, the soluble precursor $7 \mathbf{a}$ was dissolved in chlorobenzene and the solution was thoroughly degassed. This solution was heated at $200^{\circ} \mathrm{C}$ for 10 minutes and the formation of a dark violet precipitate was observed. This suspension was drop-casted on an indium-tin oxide (ITO) glass slide without any matrix and dried. In a parallel experiment, the suspension was mixed with 2,5-dihydroxy benzoic acid as a matrix and then drop-casted on an ITO glass slide and dried. These ITO glass slides were introduced in a MALDI chamber, and the high-resolution mass spectra (HRMS) were recorded. Both spectra showed only the parent peak corresponding to the nonacene $\mathbf{1}$, and no trace of a dimer as was observed for heptacene. ${ }^{30}$ These results suggest that, in contrast with heptacene, nonacene $\mathbf{1}$ is so highly insoluble that just after decarbonylation, immediate precipitation of the monomer prevents the dimerization in solution.

\section{Summary and outlook}

In summary, pure nonacene can be prepared by thermal bis-decarbonylation of precursors either in the solid state, or in high boiling point solvents. This long acene is surprisingly thermally stable up to $450^{\circ} \mathrm{C}$ and does not decompose at room temperature under dry argon. Our preparation procedure could as well be applied for the construction of substituted nonacenes and to even longer acenes, opening the way to OFETs and molecular spintronics applications.

\section{Methods}

Direct synthesis of $\mathbf{6 a} / \mathbf{6 b}$. A well-dried Schlenk flask was charged with diene 2 (200 mg, $0.72 \mathrm{mmol})$ and CsF (480 mg, $3.16 \mathrm{mmol}, 4.0$ equiv.) under argon and then anhydrous acetonitrile $(16 \mathrm{~mL})$ was added. The heterogenous mixture was cooled to $0^{\circ} \mathrm{C}$ and then solution of aryne precursor bis(trimethylsilyl)-1,4-phenylene bis(trifluoromethanesulfonate) $(298 \mathrm{mg}, 0.58 \mathrm{mmol}, 0.8$ equiv.) in anhydrous THF (4 mL) was added dropwise. The reaction was allowed to warm to room temperature overnight. Progress of the reaction was controlled by TLC, eluent (hexane - EtOAc 3:2). Reaction time depending on a scale of the reaction (1-3 days). After the evaporation of the solvent, the residue was chromatographed on silica gel (hexane : acetone 3:1) to get the desired product as a mixture of two regioisomers as a colourless solid. This mixture (146 mg, $0.23 \mathrm{mmol}) \mathrm{was}$ dissolved in anhydrous toluene $(15 \mathrm{~mL})$ under argon. The solution was cooled to $0^{\circ} \mathrm{C}$ and then DDQ (53 mg, $0.231 \mathrm{mmol}, 2$ equiv.) was added in one portion. The reaction mixture was stirred at $0^{\circ} \mathrm{C}$ for $10 \mathrm{~min}$. and then 6 hours at room temperature. The volume of the reaction mixture was reduced to a half and the mixture was filtered over a frita S4. The solid was washed with toluene and finally with methanol to get the first isomer $6 \mathrm{~b}$ $(40 \mathrm{mg})$ as a white solid. The mother liquor was evaporated and the residue was purified by chromatography on silica gel (hexane : acetone 3:1) to get the second isomer $6 \mathrm{a}(82 \mathrm{mg})$ as a white solid. The ratio of isomers is $1: 2$ and combined yield is $54 \%$ after two synthetic steps.

6a: ${ }^{1} \mathrm{H}$ NMR $\left(500 \mathrm{MHz}, \mathrm{CD}_{2} \mathrm{Cl}_{2}\right): 3.23(6 \mathrm{H}, \mathrm{s}), 3.26(6 \mathrm{H}, \mathrm{s}), 4.71(4 \mathrm{H}, \mathrm{s}), 7.33-7.36(4 \mathrm{H}, \mathrm{m}), 7.68-7.71(4 \mathrm{H}$, m), $7.70(4 \mathrm{H}, \mathrm{s}), 7.77(4 \mathrm{H}, \mathrm{s}), 8.12(2 \mathrm{H}, \mathrm{s}) \mathrm{ppm}$.

${ }^{13} \mathrm{C}$ NMR (126 MHz, $\left.\mathrm{CD}_{2} \mathrm{Cl}_{2}\right): 51.54,51.58,55.20,120.43,120.87,124.77,125.96,126.04,128.14,131.39$, $133.08,142.94,143.61 \mathrm{ppm}$.

DCI MS: $627([\mathrm{M}+\mathrm{H}]+)$.

HR DCI MS: calcd for $\mathrm{C}_{44} \mathrm{H}_{35} \mathrm{O}_{4} 627.2530$; found 627.2511 .

6b: ${ }^{1} \mathrm{H}$ NMR (300 MHz, $\left.\mathrm{CD}_{2} \mathrm{Cl}_{2}\right): 3.21(6 \mathrm{H}, \mathrm{s}), 3.22(6 \mathrm{H}, \mathrm{s}), 4.71(4 \mathrm{H}, \mathrm{s}), 7.37-7.40(4 \mathrm{H}, \mathrm{m}), 7.72-7.77(4 \mathrm{H}$, m), $7.73(4 \mathrm{H}, \mathrm{s}), 7.77(4 \mathrm{H}, \mathrm{s}), 8.12(2 \mathrm{H}, \mathrm{s}) \mathrm{ppm}$.

${ }^{13} \mathrm{C}$ NMR $\left(126 \mathrm{MHz}, \mathrm{CD}_{2} \mathrm{Cl}_{2}\right)$ : not measured due to the low solubility

DCI MS: $627\left([\mathrm{M}+\mathrm{H}]^{+}\right)$.

HR DCI MS: calcd for $\mathrm{C}_{44} \mathrm{H}_{35} \mathrm{O}_{4} 627.2530$; found 627.2523.

Synthesis of 7a: In a well dried Schlenk flask, $6 \mathbf{a}(160 \mathrm{mg}, 0.255 \mathrm{mmol})$ was dissolved in anhydrous dichloromethane $(10 \mathrm{~mL})$ under argon. Then trimethylsilyl iodide $(109 \mu \mathrm{L}, 0.766 \mathrm{mmol}, 3$ equiv. $)$ was added dropwise and the homogeneous reaction mixture was stirred overnight at room temperature. Next day the heterogeneous reaction mixture was stirred on air at room temperature for 6 hours to complete the hydrolysis of formed iodo-methoxy intermediate. The product was collected by filtration over a glass frit filter S4, washed with mixture of solvents (hexane : dichloromethane, 4:1) to afford first portion of pure compound $\mathbf{7 a}$ as a white 
solid. The mother liquor was evaporated and the residue was purified by chromatography on silica gel (hexane : acetone 3:1) to get the second portion of the product as a white solid. The combined yield was (130 $\mathrm{mg}, 95 \%)$. ${ }^{1} \mathrm{H}$ NMR (500 MHz, $\left.\mathrm{CD}_{2} \mathrm{Cl}_{2}\right): 4.99(4 \mathrm{H}, \mathrm{s}), 7.41-7.44(4 \mathrm{H}, \mathrm{m}), 7.78-7.81(4 \mathrm{H}, \mathrm{m}), 7.93(4 \mathrm{H}, \mathrm{s}), 8.04(4 \mathrm{H}, \mathrm{s})$, $8.36(2 \mathrm{H}, \mathrm{s})$.

${ }^{13} \mathrm{C}$ NMR (126 MHz, $\mathrm{CD}_{2} \mathrm{Cl}_{2}$ ): 57.55, 120.88, 121.22, 126.57, 126.76, 128.34, 131.61, 133.31, 137.61, 137.93, 194.46 ppm.

CP MAS: 56.99 (bridgehead), 120.20-137.12 (aromatic), 193.26 and $197.73(\mathrm{C}=\mathrm{O}) \mathrm{ppm}$.

DCI MS: $478\left(\left[\mathrm{M}-2 \times \mathrm{CO}^{+}\right)\right.$.

HR DCI MS: calcd for $\mathrm{C}_{38} \mathrm{H}_{22} 478.1722$ (M - 2xCO); found 478.1727.

Synthesis of 7b: In a well dried Schlenk flask, $6 \mathbf{b}(25 \mathrm{mg}, 39.89 \mu \mathrm{mol})$ was suspended in anhydrous dichloromethane $(4 \mathrm{~mL})$ under argon. Then trimethylsilyl iodide $(23 \mu \mathrm{L}, 159.6 \mu \mathrm{mol}, 4$ equiv.) was added dropwise and the heterogenous reaction mixture was stirred overnight at room temperature. Next day the heterogeneous reaction mixture was stirred on air at room temperature for 6 hours to complete the hydrolysis of formed iodo-methoxy intermediate. The product was collected by filtration over a glass frit filter S4, washed with mixture of solvents (dichloromethane : acetone, 4:1) to afford pure compound $7 \mathbf{b}(20 \mathrm{mg}, 94 \%)$ as a white solid.

CP MAS: 55.83 (bridgehead), 119.23-136.01 (aromatic), $195.55(\mathrm{C}=\mathrm{O}) \mathrm{ppm}$.

DCI MS: $478\left(\left[\mathrm{M}-2 \times \mathrm{CO}^{+}\right)\right.$.

HR DCI MS: calcd for $\mathrm{C}_{38} \mathrm{H}_{22} 478.1722$ (M - 2xCO); found 478.1731.

\section{Preparation of Nonacene 1}

Nonacene was obtained by heating $7 \mathbf{a}$ or $\mathbf{7 b}$ in the solid state at $350^{\circ} \mathrm{C}$ under vacuum for 15 min or as a suspension by heating a purged solution of $7 \mathrm{a}$ in chlorobenzene $10 \mathrm{~min}$. at $200^{\circ} \mathrm{C}$

CP MAS: 125.5 - 128.8 ppm

\section{Acknowledgements:}

The authors thank Yannick Coppel (LCC-Toulouse) for recording CP-MAS spectra, David Neumeyer (CEMES) for recording TGA spectra, Christian Bourgerette (CEMES) and Isabelle Seguy (LAAS) for help in spin-coating experiments. We gratefully acknowledge Nathalie Saffon, from the Institut de Chimie de Toulouse, who solved the X-ray structure. Vladimír Vrkoslav (IOCB, Prague) is gratefully acknowledged for the MALDI experiments. Colin Martin (NAIST-CEMES) is thanked for comments and corrections of the manuscript. A. J. acknowledges funding from the Foundation EXPERIENTIA and from ERDF/ESF "UOCHB MSCA Mobility" (No. CZ.02.2.69/0.0/ 0.0/17 050/0008490). This research has received funding from the EraNET Cofund Initiatives QuantERA under the European Union's Horizon 2020 research and innovation programme grant agreement ORQUID. This project has received financial support from the CNRS through the MITI interdisciplinary programs and JSTERATO (No. JPMJER1903) and JSPS-WPI.

\section{Author contributions}

A.J. and J.H. performed the syntheses and characterization of all products. M.S realized all the MALDI experiments. Y.N. did the simulations and calculations. A.J. and A.G designed and supervised the project.

\section{Competing interests}

The authors declare no competing interests. 


\section{References}

1. Dorel, R. \& Echavarren, A. M. Strategies for the Synthesis of Higher Acenes. European Journal of Organic Chemistry 2017, 14-24 (2016).

2. Korytár, R., Xenioti, D., Schmitteckert, P., Alouani, M. \& Evers, F. Signature of the Dirac cone in the properties of linear oligoacenes. Nature Communications 5, 5000 (2014).

3. Schmitteckert, P., Thomale, R., Korytár, R. \& Evers, F. Incommensurate quantum-size oscillations in acene-based molecular wires-Effects of quantum fluctuations. The Journal of Chemical Physics 146, 092320 (2017).

4. Krüger, J. et al. Electronic Resonances and Gap Stabilization of Higher Acenes on a Gold Surface. ACS Nano 12, 8506-8511 (2018).

5. Plasser, F. et al. The Multiradical Character of One- and Two-Dimensional Graphene Nanoribbons. Angewandte Chemie International Edition 52, 2581-2584 (2013).

6. Hachmann, J., Dorando, J. J., Avilés, M. \& Chan, G. K.-L. The radical character of the acenes: A density matrix renormalization group study. The Journal of Chemical Physics 127, 134309 (2007).

7. Jiang, D. \& Dai, S. Electronic Ground State of Higher Acenes. The Journal of Physical Chemistry A 112, 332-335 (2008).

8. Malrieu, J.-P. \& Trinquier, G. Can a Topological Approach Predict Spin-Symmetry Breaking in Conjugated Hydrocarbons? J. Phys. Chem. A 120, 9564-9578 (2016).

9. Yang, Y., Davidson, E. R. \& Yang, W. Nature of ground and electronic excited states of higher acenes. Proc Natl Acad Sci USA 113, E5098 (2016).

10. Fujita, M., Wakabayashi, K., Nakada, K. \& Kusakabe, K. Peculiar Localized State at Zigzag Graphite Edge. Journal of the Physical Society of Japan 65, 1920-1923 (1996).

11. Ruffieux, P. et al. On-surface synthesis of graphene nanoribbons with zigzag edge topology. Nature 531, 489-492 (2016). 
12. Mondal, R., Adhikari, R. M., Shah, B. K. \& Neckers, D. C. Revisiting the Stability of Hexacenes. Org. Lett. 9, 2505-2508 (2007).

13. Mondal, R., Shah, B. K. \& Neckers, D. C. Photogeneration of Heptacene in a Polymer Matrix. J. Am. Chem. Soc. 128, 9612-9613 (2006).

14. Bettinger, H. F., Mondal, R. \& Neckers, D. C. Stable photoinduced charge separation in heptacene. Chem. Commun. 5209-5211 (2007) doi:10.1039/B713059G.

15. Einholz, R. et al. Heptacene: Characterization in Solution, in the Solid State, and in Films. J. Am. Chem. Soc. 139, 4435-4442 (2017).

16. Mondal, R., Tönshoff, C., Khon, D., Neckers, D. C. \& Bettinger, H. F. Synthesis, Stability, and Photochemistry of Pentacene, Hexacene, and Heptacene: A Matrix Isolation Study. J. Am. Chem. Soc. 131, 14281-14289 (2009).

17. Tönshoff Christina \& Bettinger Holger F. Photogeneration of Octacene and Nonacene. Angewandte Chemie International Edition 49, 4125-4128 (2010).

18. Shen, B., Tatchen, J., Sanchez-Garcia, E. \& Bettinger, H. F. Evolution of the Optical Gap in the Acene Series: Undecacene. Angewandte Chemie International Edition 57, 1050610509 (2018).

19. Yamada, H. et al. Photochemical Synthesis of Pentacene and its Derivatives. Chemistry A European Journal 11, 6212-6220 (2005).

20. Strating, J., Zwanenburg, B., Wagenaar, A. \& Udding, A. C. Evidence for the expulsion of bis-CO from bridged $\alpha$-diketones. Tetrahedron Letters 10, 125-128 (1969).

21. Krüger, J. et al. Imaging the electronic structure of on-surface generated hexacene. Chem. Commun. 53, 1583-1586 (2017).

22. Krüger, J. et al. Decacene: On-Surface Generation. Angewandte Chemie International Edition 56, 11945-11948 (2017). 
23. Zuzak, R. et al. Nonacene Generated by On-Surface Dehydrogenation. ACS Nano 11, 9321-9329 (2017).

24. Zuzak, R. et al. Higher Acenes by On-Surface Dehydrogenation: From Heptacene to Undecacene. Angewandte Chemie International Edition 57, 10500-10505 (2018).

25. Urgel, J. I. et al. On-Surface Synthesis of Heptacene Organometallic Complexes. J. Am. Chem. Soc. 139, 11658-11661 (2017).

26. Zugermeier, M. et al. On-surface synthesis of heptacene and its interaction with a metal surface. Nanoscale 9, 12461-12469 (2017).

27. Urgel, J. I. et al. On-surface light-induced generation of higher acenes and elucidation of their open-shell character. Nature Communications 10, (2019).

28. Zade Sanjio S. \& Bendikov Michael. Heptacene and Beyond: The Longest Characterized Acenes. Angewandte Chemie International Edition 49, 4012-4015 (2010).

29. Watanabe, M. et al. The synthesis, crystal structure and charge-transport properties of hexacene. Nat Chem 4, 574-578 (2012).

30. Einholz, R. et al. Heptacene: Characterization in Solution, in the Solid State, and in Films. J. Am. Chem. Soc. 139, 4435-4442 (2017).

31. Jancarik, A., Levet, G. \& Gourdon, A. A Practical General Method for the Preparation of Long Acenes. Chemistry - A European Journal 25, 2366-2374 (2019).

32. Clar, E. \& Schoental, R. Polycyclic Hydrocarbons: Volume 1. (Springer Berlin / Heidelberg, 2013).

33. Tönshoff, C. \& Bettinger, H. F. Pushing the Limits of Acene Chemistry: The Recent Surge of Large Acenes. Chem. Eur. J. 27, 3193-3212 (2021).

34. Holec, J. et al. A Large Starphene Comprising Pentacene Branches. Angew. Chem. Int. Ed. anie.202016163 (2021) doi:10.1002/anie.202016163. 
35. Jancarik, A., Levet, G., Nguyen-Kahn, Hung, Gourdon, Andre, \& et al. In preparation. (2019).

36. Watanabe, M., Chen, K.-Y., Chang, Y. J. \& Chow, T. J. Acenes Generated from Precursors and Their Semiconducting Properties. Accounts of Chemical Research 46, 1606-1615 (2013).

37. Yoshida, S. \& Hosoya, T. The Renaissance and Bright Future of Synthetic Aryne Chemistry. Chemistry Letters 44, 1450-1460 (2015).

38. Pe na, D. Bottom-up Approaches to Nanographenes through Organic Synthesis. in Ideas in Chemistry and Molecular Sciences (ed. Pignataro, B.) 235-261 (Wiley-VCH Verlag GmbH \& Co. KGaA, 2010). doi:10.1002/9783527630554.ch11.

39. Rodríguez-Lojo, D., Peña, D., Pérez, D. \& Guitián, E. Straightforward Synthesis of Novel Acene-Based Aryne Precursors. Synlett 26, 1633-1637 (2015).

40. Gaussian 16, Revision C.01, M. J. Frisch, G. W. Trucks, H. B. Schlegel, G. E. Scuseria, M. A. Robb, J. R. Cheeseman, G. Scalmani, V. Barone, G. A. Petersson, H. Nakatsuji, X. Li, M. Caricato, A. V. Marenich, J. Bloino, B. G. Janesko, R. Gomperts, B. Mennucci, H. P. Hratchian, J. V. Ortiz, A. F. Izmaylov, J. L. Sonnenberg, D. Williams- Young, F. Ding, F. Lipparini, F. Egidi, J. Goings, B. Peng, A. Petrone, T. Henderson, D. Ranasinghe, V. G. Zakrzewski, J. Gao, N. Rega, G. Zheng, W. Liang, M. Hada, M. Ehara, K. Toyota, R. Fukuda, J. Hasegawa, M. Ishida, T. Nakajima, Y. Honda, O. Kitao, H. Nakai, T. Vreven, K. Throssell, J. A. Montgomery, Jr., J. E. Peralta, F. Ogliaro, M. J. Bearpark, J. J. Heyd, E. N. Brothers, K. N. Kudin, V. N. Staroverov, T. A. Keith, R. Kobayashi, J. Normand, K. Raghavachari, A. P. Rendell, J. C. Burant, S. S. Iyengar, J. Tomasi, M. Cossi, J. M. Millam, M. Klene, C. Adamo, R. Cammi, J. W. Ochterski, R. L. Martin, K. Morokuma, O. Farkas, J. B. Foresman, and D. J. Fox, Gaussian, Inc., Wallingford CT, 2016. 
41. Pieniazek, S. N., Clemente, F. R. \& Houk, K. N. Sources of Error in DFT Computations of $\mathrm{C} \square \mathrm{C}$ Bond Formation Thermochemistries: $\pi \rightarrow \sigma$ Transformations and Error Cancellation by DFT Methods. Angew. Chem. Int. Ed. 47, 7746-7749 (2008). 